\title{
Existence and approximation of nonlocal optimal design problems driven by parabolic equations
}

\author{
Fuensanta Andrés | Julio Muñoz $\mathbb{D}$ | Jesús Rosado
}

Departamento de Matemáticas,

Universidad de Castilla-La Mancha, Avda. Carlos III s/n, Toledo, Spain

\section{Correspondence}

Julio Muñoz, Departamento de

Matemáticas Universidad de Castilla-La

Mancha. Avda. Carlos III s/n, Toledo,

Spain.

Email: julio.munoz@uclm.es

Communicated by: T. Roubiček

\section{Funding information}

Ministerio de Economía, Industria y Competitividad (Spain), Grant/Award

Number: MTM2017-87912-P
This work is a follow-up to a series of articles by the authors where the same topic for the elliptic case is analyzed. In this article, a class of nonlocal optimal design problem driven by parabolic equations is examined. After a review of results concerning existence and uniqueness for the state equation, a detailed formulation of the nonlocal optimal design is given. The state equation is of nonlocal parabolic type, and the associated cost functional belongs to a broad class of nonlocal integrals. In the first part of the work, a general result on the existence of nonlocal optimal design is proved. The second part is devoted to analyzing the convergence of nonlocal optimal design problems toward the corresponding classical problem of optimal design. After a slight modification of the problem, either on the cost functional or by considering a new set of admissibility, the G-convergence for the state equation and, consequently, the convergence of the nonlocal optimal design problem are proved.

\section{KEYWORDS}

approximation of partial differential equations, integral equations, nonlocal parabolic equations, optimal control

MSC CLASSIFICATION

35J20; 49J22; 45A05; 46N20

\section{1 | INTRODUCTION}

One of the most meaningful aspects of the theory of optimal control is the characterization of possible minimizers as well as the minimizing sequences. In general, this task is complex, and therefore, the study is usually performed by means of some kind of relaxation or generalized formulation of the original problem. If the convergence of the relaxed problem toward the original problem could be guaranteed, then two important milestones would be realized. On one side, the generalization of the problem would lead us to consider a larger set of admissible elements, and consequently, a greater capacity to reproduce phenomena would be accomplished. This approach, which is by itself essential for the modelization, together with the fact that the set of admissibility is less strict than in the classical setting, would make the problem more tractable. On the other side, if one could assume convergence of the relaxed problem towards the original problem, then the sequences of solutions of the relaxed one could provide valuable information about the minimizing sequences of the original one.

In line with the strategy that we have just described, we define the generalized problem as the nonlocal adaptation of the original one. The problem we formulate is indeed a family of nonlocal parabolic problems depending on a parameter, 
which is usually called horizon. This parameter is the responsible for the intensity of interaction between pairs of states evaluated upon points that can be quite separate. This nonlocality is present both in the state equation and in the cost functional. For each value of the parameter, and for any admissible diffusion coefficient, the existence and uniqueness of solution for the state equation is a well-known fact. In the first part of the work, we investigate whether the optimal control (design) problem is well posed if the control function is the diffusion coefficient and the boundary conditions are Dirichlet homogeneous. In the second part of the work, once the existence of optimal designs for the nonlocal problem has been proved, we intend to explore the convergence towards the original local problem when the horizon tends to zero. In that process, the key stage is to know if the state equation gamma-converges, or G-converges, toward the state equation of the local problem. We will provide a framework for which this convergence is fulfilled. As a by-product of this study, two different proposals of regularization for the local optimal design problem are obtained.

The literature on nonlocal parabolic problems is extensive and has been used to study interesting types of modelization in different fields of applied mathematics. ${ }^{1,2}$ The nonlocality has served to build models in finance, ${ }^{3}$ fluid dynamics, ${ }^{4,5}$ elasticity, ${ }^{6-8}$ biology, ${ }^{9,10}$ image processing, ${ }^{11,12}$ or nonconvex variational analysis. ${ }^{13-15}$ In connection with the development of this sort of problems, a collection of works on analysis have participated in an essential way (see, for instance, Andreu-Vaillo et al, ${ }^{16}$ Bourgain et al,${ }^{17}$ Brezis, ${ }^{18}$ and Ponce ${ }^{19,20}$ ). Special attention and profusion in the context of diffusion have received nonlinear problems, especially the case of fractional $p$-Laplacian (see previous studies ${ }^{2,16,21,22}$ ). Also, in what concerns the numerical analysis, there are multiple contributions to facilitate the comprehension of the equations and the efficiency of different numerical schemes. ${ }^{23-29}$

However, there is not much literature concerning nonlocal optimal design models. We refer, among others, Andrés and Muñoz, ${ }^{30,31}$ Bonder and Spedaletti, ${ }^{32}$ D'Elia and Gunzburger, ${ }^{33,34}$ and Zhou and Du ${ }^{35,36}$ mainly in the context of elliptic equations. Also, Bonder et $\mathrm{al}^{37}$ is a valuable paper, where an $\mathrm{H}$-convergence study has been carried out.

\section{1 | Formulation of the problem}

The problem we analyze concerns the optimization of a given functional that is constrained to a specific type of nonlocal parabolic equation. The optimal design problem can be formulated as

$$
\min _{(h, u) \in \mathcal{A}} C(h, u)
$$

where $C(\cdot, \cdot)$ is the cost functional, and the functional space $\mathcal{A}$, which will be specified later, is characterized by the nonlocal diffusion equation that any admissible pair $(h, u)$ must satisfy

$$
\left(P_{\delta}\right) \equiv\left\{\begin{array}{l}
u_{t}(x, t)+L_{\delta}(u(x, t))=f(x, t), \text { in } \Omega, \\
u(x, t)=0 \text { in } \Omega_{\delta}-\Omega, t \in(0, T), \\
u(x, 0)=g(x) \text { in } \Omega,
\end{array}\right.
$$

where

$$
L_{\delta}(u(x, t))=-2 \int_{B(x, \delta)} \frac{k_{\delta}\left(\left|x^{\prime}-x\right|\right)}{\left|x^{\prime}-x\right|^{2}} H\left(x^{\prime}, x\right)\left(u\left(x^{\prime}, t\right)-u(x, t)\right) d x^{\prime}
$$

with $\delta$ a fixed, positive, and small parameter. In addition, the following conditions hold:

1. $\Omega$ is a bounded Lipschitz domain in $\mathbb{R}^{N}$ and $\Omega_{\delta}=\Omega \cup\left\{\cup_{p \in \partial \Omega} B(p, \delta)\right\}$ where $B(x, r)$ denotes the open ball centered at $x \in \mathbb{R}^{N}$ and radius $r>0$.

2. $g \in L^{2}\left(\Omega_{\delta}\right)$ and $f \in L^{2}\left(0, T ; L^{2}(\Omega)\right)$, where

$$
L^{2}\left(0, T ; L^{2}(\Omega)\right)=\left\{f:[0, T] \rightarrow L^{2}(\Omega): \int_{0}^{T}\|f\|_{L^{2}(\Omega)}^{2} d t<\infty\right\} .
$$

3. $H$ is defined as

with $h$ in the space

$$
H\left(x^{\prime}, x\right)=\frac{h(x)+h\left(x^{\prime}\right)}{2}
$$

$$
\mathcal{H} \doteq\left\{h: \Omega_{\delta} \rightarrow\left[h_{\min }, h_{\max }\right] \text { a.e. } x \in \Omega, h=0 \text { in } \Omega_{\delta}-\Omega \text { and } \int_{\Omega} h(x) d x=V_{0}\right\} .
$$


The constants $0<h_{\min }<h_{\max }$ are given and satisfy the constraints $h_{\min }|\Omega|<V_{0}<h_{\max }|\Omega|$.

4. For each $\delta>0$, the kernel $k_{\delta}$, satisfies

(a) $k_{\delta}$ is a non-negative radial function such that

$$
\operatorname{supp} k_{\delta} \subset B(0, \delta)
$$

and

$$
\frac{1}{N} \int_{B(0, \delta)} k_{\delta}(|s|) d s=1 .
$$

(b) The function $K_{r}: B(0, \delta)-\{0\} \rightarrow(0,+\infty)$ defined by $K_{r}(z)=\frac{k_{\delta}(|z|)}{|z|}$ is integrable

$$
K_{r} \in L^{1}(B(0, \delta)) .
$$

(c) The function $K_{s}: B(0, \delta)-\{0\} \rightarrow[0,+\infty)$ defined by $K_{S}(z)=\frac{k_{\delta}(|z|)}{|z|^{2}}$ is singular near the origin in the sense that

$$
\lim _{\theta \rightarrow 0^{+}} \int_{B(0, \delta)-B(0, \theta)} K_{s}(z) d z=+\infty
$$

In order to understand the problem, we previously need to know the spaces involved. First of all, we define $L_{0}^{2}\left(\Omega_{\delta}\right)$ as

$$
L_{0}^{2}\left(\Omega_{\delta}\right)=\left\{u: \Omega_{\delta} \rightarrow \mathbb{R}: u \in L^{2}(\Omega ; \mathbb{R}) \text { and } u=0 \text { in } \Omega_{\delta}-\Omega\right\},
$$

and the space

$$
X=\left\{v \in L_{0}^{2}\left(\Omega_{\delta}\right): B_{h}(u, u)<\infty\right\}
$$

where

$$
B_{h}(u, v)=\int_{\Omega_{\delta}} \int_{\Omega_{\delta}} H\left(x^{\prime}, x\right) \frac{k_{\delta}\left(\left|x^{\prime}-x\right|\right)}{\left|x^{\prime}-x\right|^{2}}\left(u\left(x^{\prime}, t\right)-u(x, t)\right)\left(v\left(x^{\prime}, t\right)-v(x, t)\right) d x^{\prime} d x .
$$

We also define the following subspace of $X$,

$$
X_{0}=\overline{C_{c o}^{\infty}\left(\Omega_{\delta}\right)}
$$

where

$$
C_{c o}^{\infty}\left(\Omega_{\delta}\right)=\left\{v \in C_{c}^{\infty}\left(\Omega_{\delta}\right): v=0 \text { en } \Omega_{\delta}-\Omega,\right\} \subset X .
$$

The closure is defined with respect the norm $\|\cdot\|$ given through the quadratic form $B_{h}(\cdot, \cdot)$ :

$$
X_{0}=\left\{v \in X: \exists\left(v_{j}\right) \subset C_{c o}^{\infty}\left(\Omega_{\delta}\right) \text { such that } \lim _{j} B_{h}\left(v_{j}-v, v_{j}-v\right)=0\right\} .
$$

We notice that, for each $h$ and $\delta$ fixed, both $X$ and $X_{0}$ are Hilbert spaces with the inner product $B_{h}(\cdot, \cdot)$. These spaces depends on $\delta$ but not on $h$ because the underlying norms are equivalents. ${ }^{38}$ Another important issue for each $\delta$ is the chain of dense and continuous embeddings

$$
X_{0} \subset L_{0}^{2} \subset X_{0}^{\prime}
$$

where $X_{0}^{\prime}$ denotes the dual of $X_{0}$. We are able to identify (a) $X_{0}$ with a dense subspace of $L_{0}^{2}$ (as in the classical setting $C_{c}^{\infty}(\Omega) \subset H_{0}^{1}(\Omega)$ is dense in $L^{2}(\Omega)$ ); (b) $L_{0}^{2}\left(\Omega_{\delta}\right)$ with itself, by means of its own inner product (as usual for a Hilbert space); (c) $L_{0}^{2}\left(\Omega_{\delta}\right)$ with a subspace of $X_{0}^{\prime}$, so that any function of $L_{0}^{2}\left(\Omega_{\delta}\right)$ acts on $X_{0}$ via the inner product of $L_{0}^{2}\left(\Omega_{\delta}\right)$ (Andrés ${ }^{38}$ ). Recall that the above three separable Hilbert spaces constitute what is called a Gelfand triplet (see previous studies ${ }^{39-41}$ and Appendix A).

We finally define

$$
Y_{0}=L^{2}\left(0, T ; X_{0}\right)
$$

that is, the space

$$
Y_{0}=\left\{u(., t) \in X_{0}: \int_{0}^{T}\|u\|_{X_{0}}^{2} d t<\infty\right\},
$$

which is also a Hilbert space. The solution of the problem $\left(P_{\delta}\right)$ defined in $(2)$ must be understood in a weak sense: 
Definition 1. We say that $u \in Y_{0}=L^{2}\left(0, T ; X_{0}\right)$ is a weak solution of Problem (2), if $u_{t} \in L^{2}\left(0, T ; X_{0}^{\prime}\right)$, $u$ satisfies the evolution equation of $\left(P_{\delta}\right)$, namely,

$$
\left\langle u_{t}, v\right\rangle+B_{h}(u, v)=(f(x, t), v)
$$

for any $v \in X_{0}$ and a.e. $t \in[0, T]$, and

$$
u(x, 0)=g(x), x \in \Omega .
$$

Remark 1. The derivative of $u$ with respect to $t, u_{t}$, has to be understood in a weak sense. For each $t \in[0, T]$, the solution $u$ has distributional derivative $u_{t}$ in $X_{0}^{\prime}$; here, $(\cdot, \cdot)$ denotes the usual inner product in $L^{2}(\Omega)$ while $\langle\cdot, \cdot\rangle$ stands for the pairing of $X_{0}^{\prime}$ and $X_{0}$. Henceforth, we will use this short notation, when there is no risk of misunderstanding.

Since $X_{0} \subset L_{0}^{2} \subset X_{0}^{\prime}$ is a Gelfand triplet, we are allowed to use classical results to ensure that any solution of (2), after an appropriate modification on a null set, belongs to the $C\left([0, T] ; L_{0}^{2}\left(\Omega_{\delta}\right)\right)$. Thus, the initial condition (11) makes sense. See Theorem 8 in Appendix A.

Finally, the cost functional in (1) is defined on the admissible pairs according to the formula

$$
C(h, u)=\int_{0}^{T} \int_{\Omega_{\delta}} \int_{\Omega_{\delta}} F\left(t, x, x^{\prime}, u\left(x^{\prime}, t\right), u(x, t)\right) d x^{\prime} d x d t
$$

where the integrand $F:(0, T) \times \Omega_{\delta} \times \Omega_{\delta} \times \mathbb{R} \times \mathbb{R} \rightarrow \mathbb{R}$ is assumed to verify the following conditions:

1. $F\left(\cdot, \cdot, \cdot, u_{1}, u_{2}\right)$ is a measurable function for any $\left(u_{1}, u_{2}\right) \in \mathbb{R} \times \mathbb{R}$.

2. $F\left(t, x_{1}, x_{2}, \cdot, \cdot\right)$ is a lower semicontinuous function for any $\left(t, x_{1}, x_{2}\right) \in(0, T) \times \Omega_{\delta} \times \Omega_{\delta}$.

Consequently, the optimal design problem (1)-(2) can be explicitly written as

$$
\min _{(h, u) \in \mathcal{A}} \int_{0}^{T} \int_{\Omega_{\delta}} \int_{\Omega_{\delta}} F\left(t, x, x^{\prime}, u\left(x^{\prime}, t\right), u(x, t)\right) d x^{\prime} d x d t
$$

where the set of admissibility is

$$
\mathcal{A}=\left\{\left(h, u_{\delta}\right) \in \mathcal{H} \times Y_{0}:\left(h, u_{\delta}\right) \text { satisfy }(2)\right\} .
$$

Definition 2. Fixed $\delta>0$, we say that $\left(h_{\delta}^{*}, u_{\delta}^{*}\right) \in \mathcal{A}$ is a solution of the optimal design problem (12)-(13) if

$$
C\left(h_{\delta}^{*}, u_{\delta}^{*}\right) \leq C(h, u)
$$

for any $(h, u) \in \mathcal{A}$.

\section{2 | Contributions and organization of the paper}

Section 2 is a series of statements indispensable to understand the techniques we have employed. In Section 2.1, the basics about the spectral analysis of nonlocal the elliptic operators and the existence of solution for the nonlocal parabolic equations is recalled. Section 2.2 states the convergence of the solution of the nonlocal parabolic problem towards the solution of the corresponding local parabolic equation, when the horizon tends to zero. In a third part of Section 2, we recall one of our key points of these research; Theorem 1 is a continuity result of the spectra with respect to the controls. In addition, and for the sake of clarity, Section 2.4 contains two compactness results that have been essential in our research, and they will be used again in this article (Theorems 2 and 3). All details for the derivation of these achievements can be found at papers..$^{30,31,38,42,43}$

The main result of this paper is the existence of nonlocal optimal design (Theorem 4). The proof in given in Section 3. Next, Section 4 is devoted to the convergence of the sequence of the optimal designs. The aim is to build some particular nonlocal design problems for which the existence of nonlocal optimal controls is ensured and such that, in the limit, these controls converge to the solution of a local design problem (Theorems 6 and 7). These results previously require a G-convergence result for the nonlocal parabolic equation (Theorem 5). 


\section{2 | PRELIMINARIES}

In this section, we recall some essential results.

\section{1 | Spectral analysis and existence of solution for the nonlocal parabolic equation}

The first issue we must underline is the spectral analysis of the nonlocal operators $B_{h}$. The main result is the existence of a basis of eigenfunctions $\left\{w_{\delta}^{(k)}\right\} \subset X_{0}$, with eigenvalues $\gamma_{k}^{n l}(\delta)$ for the nonlocal operator $B_{h_{\delta}}$, so that for any $v \in X_{0}$, we can write

$$
B_{h}\left(w_{\delta}^{(k)}, v\right)=\gamma_{k}^{n l}(\delta)\left(w_{\delta}^{(k)}, v\right)_{L^{2}(\Omega) \times L^{2}(\Omega)} .
$$

The sequence of eigenvalues $\gamma_{k}^{n l}(\delta)$ is nondecreasing and $\gamma_{k}^{n l}(\delta) \rightarrow+\infty$ when $k \rightarrow+\infty$. Concerning the eigenfunctions, we know $\left\{w_{\delta}^{(k)}\right\}$ is an orthonormal basis in $L_{0}^{2}\left(\Omega_{\delta}\right)$ and orthogonal in $X_{0}$. The proof of these claims directly follows from basics tools of linear algebra and makes use of the compactness embedding $X_{0} \subset L_{0}^{2}\left(\Omega_{\delta}\right)$ (see Theorem 2 below). Consult Andrés, ${ }^{38}$ Andrés and Muñoz, ${ }^{42}$ and Servadei and Valdinoci ${ }^{44}$ for a detailed analysis.

The described framework and the above spectral results provide the appropriate tool to solve the problem (2): the Galerkin-Fourier method. We know that for a given $h \in \mathcal{H}$ and a $\delta>0$ fixed, there exists a unique solution $u_{\delta} \in Y_{0}$ of Equation (2). Moreover, if $f(x, t)=\sum_{k=1}^{\infty} f_{\delta k}(t) w_{\delta}^{(k)}(x)$, where $\left(f_{\delta k}\right)_{k}$ is the sequence of Fourier coefficients given by $f_{\delta k}(t)=\int_{\Omega} f(x, t) w_{\delta}^{(k)}(x) d x$, and $\left(g_{\delta k}\right)_{k}$ is the sequence of Fourier coefficients of $g$, then

$$
u_{\delta}(x)=\sum_{k=1}^{\infty} d_{\delta}^{(k)}(t) w_{\delta}^{(k)}(x)
$$

where $d_{\delta}^{(k)}$ is the solution to the initial value problem

$$
\left\{\begin{array}{c}
\left(d_{\delta}^{(k)}(t)\right)^{\prime}+\gamma_{k}^{n l}(\delta) d_{\delta}^{(k)}(t)-f_{\delta k}(t)=0 \\
d_{\delta}^{(k)}(0)=g_{\delta k}
\end{array}\right.
$$

Again, the details of all these results can be consulted in Andrés, ${ }^{38}$ Andrés and Muñoz, ${ }^{42}$ and Servadei and Valdinoci. ${ }^{44}$

\subsection{Convegence to the local problem}

Next, a relevant aspect that we must have in mind is the asymptotic behavior of the sequence of solutions $\left(u_{\delta}\right)_{\delta}$ when $\delta \rightarrow 0$ with $h \in \mathcal{H}$. Consequently, some notes related to the local problem are in order. The classical or local problem is formulated as follows: Find $u \in W(0, T)$ such that

$$
(P) \equiv\left\{\begin{array}{l}
w_{t}(x, t)-\operatorname{div}(h(x) w(x, t))=f(x, t), \text { in } \Omega, \\
w(x, t)=0 \text { in } \partial \Omega, \\
w(x, 0)=g(x)
\end{array}\right.
$$

The space $W(0, T)$ is a Hilbert space described as

$$
W(0, T)=\left\{v \in L^{2}\left(0, T ; H_{0}^{1}(\Omega)\right): v_{t} \in L^{2}\left(0, T ; H^{-1}(\Omega)\right)\right\}
$$

and whose inner product is given by

$$
(u, v)=\int_{0}^{T}\left(\nabla_{x} u(x, t), \nabla_{x} v(x, t)\right)_{L^{2}(\Omega)} d t+\int_{0}^{T}\left\langle u_{t}(x, t), v_{t}(x, t)\right\rangle_{H^{-1}(\Omega)} d t .
$$

In this framework, it is known the following result of convergence: From the sequence of solutions of $(2),\left(u_{\delta}\right)_{\delta}$, we can extract a subsequence that converges strongly in $Y_{0}$ to a function $u \in W(0, T)$ when $\delta \rightarrow 0$. Moreover, $u$ is the weak solution of the local problem (16). We refer the reader to previous works, ${ }^{38,43,45,46}$ for instance, for the details concerning this kind of nonlocal convergence. 


\subsection{Convergence of the spectra}

The intrinsic structure of the optimal design problem we have just described lies also in the convergence of the spectra. In this case, the asymptotic is not with respect to the horizon parameter $\delta$, it has to do with the limit when we consider a minimizing sequence.

Let $\left(h_{j}\right)_{j}$ be a sequence of admissible designs. From this sequence, we extract a subsequence whose weak-* limit in $L^{\infty}\left(\Omega_{\delta}\right)$ is $h$. Then, with $t$ and $\delta$ fixed, and for each $j$, as we have already indicated, the operator $B_{h_{j}}$ has a unitary basis of eigenfunctions. The notation is $\gamma_{1}^{n l}(j)$ is the first eigenvalue and $w_{j}^{(1)}$ its corresponding eigenfunction. For $k \geq 2$, the eigenvalue is $\gamma_{k}^{n l}(j)$ and its corresponding set of eigenfunctions $\cup_{i=1}^{n_{k}} w_{i j}^{(k)}\left(n_{k} \in \mathbb{N}\right)$. In the same way for $B_{h}$, we assume its first eigenfunction is $\psi^{(1)}$ with eigenvalue $\gamma_{1}^{n l}$; and for $k \geq 2$, the set $\cup_{i=1}^{l_{k}} \psi_{i}^{(k)}$ will denote the set of eigenvector whose eigenvalue is $\gamma_{k}^{n l}$.

Under these circumstances, we have

Theorem 1 (Andrés and Muñoz ${ }^{30}$ ).

1. Concerning the first eigenvalue, $\gamma_{1}^{n l}(j)$, and the first eigenfunction $w_{j}^{(1)}\left(l_{1}=1\right)$, there is a subsequence of indexes $j$ still denoted by $j$ such that $\lim _{j} \gamma_{1}^{n l}(j)=\gamma_{1}$ and $\lim _{j} w_{j}^{(1)}=\psi^{(1)}$ in $L^{2}\left(\Omega_{\delta}\right)$.

2. For each $k \geq 2$, we have $n_{k}=l_{k}$, and there is a subsequence of indexes $j$, still denoted by $j$, such that $\lim _{j} \gamma_{k}^{n l}(j)=\gamma_{k}$ and a set of functions $\cup_{i=1}^{n_{k}} w_{i}^{(k)}$ in $X_{0}$ such that $\lim _{j} w_{i j}^{(k)}=w_{i}^{(k)}$ strong in $L^{2}$, where $w_{i}^{(k)}$ are eigenfunctions for the nonlocal problem defined by $B_{h}$ with eigenvalues $\gamma_{k}$, for $i=1, \ldots, n_{k}$. Moreover $\mathcal{L}\left\{\cup_{i=1}^{n_{k}} w_{i}^{(k)}\right\}=\mathcal{L}\left\{\cup_{i=1}^{n_{k}} \psi_{i}^{(k)}\right\}$ for any $k$.

\section{4 | Compactness results}

The proof of Theorem 1 straightforwardly follows from some essential results by Brezis, Ponce and other authors. In particular, it makes use of these two interesting compactness tools:

Theorem 2. For each $\delta>0$ and $h \in \mathcal{H}$ fixed.

1. (Andreu-Vaillo et al, ${ }^{16}$ Ponce, ${ }^{19}$ Andrés and Muñoz ${ }^{42}$ ) There is a positive constant $A_{p}$, depending only on $\Omega$ such that for every $\psi \in X_{0}$,

$$
B_{h}(\psi, \psi) \geq A_{p}\|\psi\|_{L^{2}(\Omega)}^{2}
$$

(nonlocal Poincaré inequality).

2. (Andrés and Muñoz $z^{31,42}$ ) For any given constant $C>0$, the set

$$
\mathcal{X}_{C}(\delta)=\left\{\psi \in L_{0}^{2}\left(\Omega_{\delta}\right): B_{h}(\psi, \psi)<C\right\}
$$

is compactly imbedded in $L_{0}^{2}\left(\Omega_{\delta}\right)$.

Theorem 3 (Bourgain et al, ${ }^{17}$ Brezis, ${ }^{18}$ Ponce, ${ }^{19}$ and Andrés and Muñoz $\left.{ }^{42}\right)$. If $\left(\psi_{\delta}\right)_{\delta}$ is a bounded sequence in $L_{0}^{2}\left(\Omega_{\delta}\right)$ and there is a positive constant $C$ such that $B_{h}\left(\psi_{\delta}, \psi_{\delta}\right) \leq C$, for any $\delta \leq \delta_{0}$, then $\left(\psi_{\delta}\right)_{\delta}$ is relatively compact in $L_{0}^{2}\left(\Omega_{\delta}\right)$. Moreover, if $\left(\psi_{\delta_{j}}\right)_{j}$ is a subsequence such that $\psi_{\delta_{j}} \rightarrow \psi$ strong in $L^{2}(\Omega)$, if $j \rightarrow+\infty$, then $\psi \in H^{1}(\Omega)$.

\section{3 | EXISTENCE OF OPTIMAL DESIGNS}

The aim is to proof the following result:

Theorem 4. There exists at least a pair $(h, u) \in \mathcal{H} \times Y_{0}$ solution to the problem (12)-(13).

We base the idea of the proof on some key asymptotic results. 


\section{1 | Step 1}

Assume the parameter $\delta>0$ is fixed and let $\left(h_{j}, u_{j}\right)_{j}$ be a minimizing sequence of the problem (12)-(13). Then

$$
\left\langle\left(u_{j}\right)_{t}, u_{j}\right\rangle+B_{h_{j}}\left(u_{j}, u_{j}\right)=\left(f, u_{j}\right)_{L^{2}(\Omega) \times L^{2}(\Omega)} .
$$

As in the local case, the use of the above variational equation, Theorem 8, and Poincaré inequality (17) yield

$$
\begin{aligned}
A_{p} \int_{0}^{T}\left\|u_{j}\right\|_{L^{2}\left(\Omega_{\delta}\right)}^{2}(t) d t & \leq \int_{0}^{T} B_{h_{j}}\left(u_{j}, u_{j}\right) d t \\
& \leq \frac{1}{2}\|g\|_{L^{2}(\Omega)}^{2}+\left(\int_{0}^{T}\|f\|_{L^{2}(\Omega)}^{2} d t\right)^{1 / 2}\left(\int_{0}^{T}\left\|u_{j}\right\|_{L^{2}(\Omega)}^{2} d t\right)^{1 / 2} .
\end{aligned}
$$

This clearly implies that $\int_{0}^{T}\left\|u_{j}\right\|_{L^{2}\left(\Omega_{\delta}\right)}^{2}(t) d t$ is bounded, and consequently, $\int_{0}^{T} B_{h_{j}}\left(u_{j}, u_{j}\right) d t$ too.

Now, we shall prove uniform estimations:

Proposition 1. The sequences $\left\|u_{j}\right\|_{L^{2}\left(\Omega_{\delta}\right)}^{2}$ and $B_{h_{j}}\left(u_{j}, u_{j}\right)$ are uniformly bounded in $j$, for a.e. $t \in(0, T]$.

Proof. By Poincaré inequality (17), it suffices to prove

$$
B_{h_{j}}\left(u_{j}, u_{j}\right)<C,
$$

for any $j$. Thanks to (14)-(15), we can write

$$
u_{j}(x, t)=\sum_{k=1}^{\infty} d_{j}^{(k)}(t) w_{j}^{(k)}(x)
$$

where

$$
d_{j}^{(k)}(t)=\int_{0}^{t} f_{j}^{(k)}(s) \exp \left(\gamma_{j k}^{n l}(s-t)\right) d s+g_{j}^{(k)} \exp \left(-\gamma_{j k}^{n l} t\right) .
$$

By using the above expansion of $u_{j}$ and Hölder inequality, we get

$$
\begin{aligned}
B_{h_{j}}\left(u_{j}(x, t), u_{j}(x, t)\right)= & \sum_{k=1}^{\infty}\left(d_{j}^{(k)}(t)\right)^{2} \gamma_{j k}^{n l} \\
\leq & \sum_{k=1}^{\infty} \int_{0}^{t}\left(f_{j}^{(k)}(s)\right)^{2} d s\left(2 \gamma_{j k}^{n l} \int_{0}^{t} \exp \left(2 \gamma_{j k}^{n l}(s-t)\right) d s\right) \\
& +\sum_{k=1}^{\infty}\left(g_{j}^{(k)}\right)^{2} 2 \gamma_{j k}^{n l} \exp \left(-2 \gamma_{j k}^{n l} t\right) \\
\leq & \sum_{k=1}^{\infty} \int_{0}^{T}\left(f_{j}^{(k)}(s)\right)^{2} d s+\frac{1}{t} \sum_{k=1}^{\infty}\left(g_{j}^{(k)}\right)^{2},
\end{aligned}
$$

whence we obviously deduce (19).

In view of the above discussion, Theorem 2 ensures the sequence $\left(u_{j}\right)_{j}$ is precompact in $L^{2}\left(\Omega_{\delta}\right)$ : Hence, there is $u(\cdot, t) \in$ $L^{2}\left(\Omega_{\delta}\right)$ such that for a subsequence of $\left(u_{j}\right)_{j}$,

$$
u_{j} \rightarrow u \text { strongly in } L^{2}\left(\Omega_{\delta}\right) .
$$

Also, since

$$
\int_{0}^{T} B_{h_{j}}\left(u_{j}, u_{j}\right) d t=\int_{0}^{T}\left\|u_{j}\right\|_{X_{0}}^{2} d t \leq C
$$


then

$$
u_{j} \rightarrow u \text { weakly in } Y_{0} .
$$

\section{2 | Step 2}

We are going to see the pair $(h, u)$, where $u$ is the limit of the sequence $\left(u_{j}\right)_{j}$ described asbove and $h$ is the weak-* limit in $L^{\infty}$ of the sequence $\left(h_{j}\right)_{j}$, is admissible. Since $u \in Y_{0}$ and $h \in \mathcal{H}$, it only remains to see whether they fulfill the nonlocal state equation.

By Theorem 1, we know

$$
\lim _{j \rightarrow+\infty} \gamma_{k}^{n l}(j)=\gamma_{k}^{n l}
$$

and

$$
\lim _{j \rightarrow+\infty} w_{i j}^{(k)}(x)=w_{i}^{(k)}(x) \text { strong in } L^{2} \text {, for } i=1, \ldots, n_{k},
$$

where $\gamma_{k}^{n l}(j)$ are the eigenvalues of the eigenfunctions $w_{i j}^{(k)}(x)$ for $i=1, \ldots, n_{k}$, of the nonlocal operator $B_{h_{i}}$. In the same way, $\gamma_{k}^{n l}$ are the eigenvalues that correspond to the eigenfunctions $w_{i}^{(k)}(x)$, for $i=1, \ldots, n_{k}$, of the nonlocal operator $B_{h}$. We also recall $\cup_{k=1}^{\infty} \cup_{i=1}^{n_{k}} w_{i j}^{(k)}(x)$ and $\cup_{k=1}^{\infty} \cup_{i=1}^{n_{k}} w_{i}^{(k)}(x)$ are orthogonal basis in $X_{0}$. From these assertions, we easily deduce

$$
\begin{aligned}
\lim _{j \rightarrow+\infty} f_{i j}^{(k)}(t) & =\lim _{j \rightarrow+\infty}\left(f, w_{i j}^{(k)}\right)=\left(f, w_{i}^{(k)}\right)=f_{i}^{(k)}, \\
\lim _{j \rightarrow \infty} g_{i j}^{(k)} & =\lim _{j \rightarrow+\infty}\left(g, w_{i j}^{(k)}\right)=\left(g, w_{i}^{(k)}\right)=g_{i}^{(k)}
\end{aligned}
$$

for $i=1, \ldots, n_{k}$. Thanks to the above limits, we arrive at

$$
\lim _{j \rightarrow \infty} u_{i j}^{(k)}=\lim _{j \rightarrow \infty}\left(u_{j}, w_{i j}^{(k)}(x)\right)=\left(u, w_{i}^{(k)}\right)=u_{i}^{(k)}
$$

for $i=1, \ldots, n_{k}$, and thus,

$$
\begin{aligned}
u_{i}^{(k)} & =\lim _{j \rightarrow \infty} u_{i j}^{(k)}=\lim _{j \rightarrow \infty} d_{i j}^{(k)}(t) \\
& =\lim _{j \rightarrow \infty} \int_{0}^{t} f_{i j}^{(k)}(s) \exp \left(\gamma_{k}^{n l}(j)(s-t)\right) d s+g_{i j}^{(k)} \exp \left(-\gamma_{k}^{n l}(j) t\right) \\
& =\int_{0}^{t} f_{i}^{(k)}(s) \exp \left(\gamma_{k}^{n l}(s-t)\right) d s+g_{i}^{(k)} \exp \left(-\gamma_{k}^{n l} t\right)=d_{i}^{(k)}(t)
\end{aligned}
$$

for $i=1, \ldots, n_{k}$, where $f_{i}^{(k)}(t)=\int_{\Omega} f(x, t) w_{i}^{(k)}(x) d x$ and $g_{i}^{(k)}=\int_{\Omega} g(x) w_{i}^{(k)}(x) d x$. If we omit the multiplicity in the notation for the eigenfunctions, we expand the limit $u$ in the basis $\cup_{k=1}^{\infty} \cup_{i=1}^{n_{k}} w_{i}^{(k)}(x)$, and we use the above results of convergence, then we can write

$$
\begin{aligned}
u(x, t) & =\sum_{k=1}^{\infty} d_{k}(t) w^{(k)}(x) \\
& =\sum_{k=1}^{\infty}\left(\int_{0}^{t} f^{(k)}(s) \exp \left(\gamma_{k}(s-t)\right) d s+g^{(k)} \exp \left(-\gamma_{k} t\right)\right) w^{(k)}(x)
\end{aligned}
$$

Moreover, this function $u$ is the solution of (10) (see Lions ${ }^{47}$ or Evans ${ }^{48}$ ).

As a consequence of the above process, we state the convergence

$$
\lim _{j \rightarrow \infty} B_{h_{j}}\left(u_{j}, u_{j}\right)=B_{h}(u, u)
$$

\section{3 | Step 3}

We are going to prove the pair $(h, u)$ is a solution of (12)-(13). This admissible pair has been obtained as the limit of the minimizing sequence $\left(h_{j}, u_{j}\right)$ in the sense that $h$ is the weak-* limit in $L^{\infty}$ of $\left(h_{j}\right)_{j}$, and $u$ is both the weak limit in $Y_{0}$ and 
the strong limit in $L^{2}$ of the sequence $\left(u_{j}\right)_{j}$. Then, we can apply Fatou lemma, if it is necessary for a subsequence, to get

$$
\begin{aligned}
& \lim _{j} \int_{0}^{T} \int_{\Omega_{\delta}} \int_{\Omega_{\delta}} F\left(t, x, x^{\prime}, u_{j}\left(x^{\prime}, t\right), u_{j}(x, t)\right) d x^{\prime} d x d t \\
& \geq \int_{0}^{T} \int_{\Omega_{\delta}} \int_{\Omega_{\delta}} F\left(t, x, x^{\prime}, u\left(x^{\prime}, t\right), u(x, t)\right) d x^{\prime} d x d t,
\end{aligned}
$$

which serves to ensure the pair $(h, u)$ is a solution of the local design problem.

\section{CONVERGENCE OF A CLASS OF NONLOCAL OPTIMAL DESIGN PROBLEMS}

The motivation is the extension to the parabolic case of some results from Andrés and Muñoz. ${ }^{31}$ The goal is to produce some results that could help us to approximate the solutions of the following local problem $\left(D_{0}\right)$ :

$$
\min _{(h, u) \in \mathcal{A}} \int_{0}^{T} \int_{\Omega}|\nabla u(x, t)|^{2} d x d t
$$

where

$$
\mathcal{A}_{\text {loc }}=\{(h, u) \in \mathcal{H} \times W(0, T):(h, u) \text { satisfy }(16)\} .
$$

The idea is to work with a nonlocal optimal design problem $\left(\mathcal{D}_{\delta}\right)$ for which we know existence of optimal designs $\left(h_{\delta}, u_{\delta}\right)\left(h_{\delta}\right.$ the design and $u_{\delta}$ its associated state) and then pass to the limit in order to recover a local problem that could be interpreted as a regularization of the problem $\left(D_{l o c}\right)$. We give a more precise explanation of that.

\section{1 | Description of the problem}

\subsection{1 | The nonlocal model}

The aim is to analyze the asymptotic when $\delta \rightarrow 0$ of some kind of optimal design problems. The first type of problem we are going to discuss is $\left(\mathcal{D}_{\delta}\right)$; it is defined as follows:

$$
\min _{(h, u) \in \mathcal{A}} J_{\delta}
$$

where the cost functional is

$$
J_{\delta}(h, u)=\int_{0}^{T} B(u, u) d t+c B_{0}(h, h)
$$

with

$$
B_{0}(h, h)=\int_{\Omega_{\delta_{0}}} \int_{\Omega_{\delta_{0}}} k_{\delta_{0}}\left(\left|x^{\prime}-x\right|\right) \frac{\left|h\left(x^{\prime}\right)-h(x)\right|^{2}}{\left|x^{\prime}-x\right|^{2}} d x^{\prime} d x,
$$

and $\delta_{0}>0$ and $c \geq 0$ are given constants. The set of admissibility is

$$
\mathcal{A}=\left\{(h, u) \in \mathcal{H} \times Y_{0}:(h, u) \operatorname{satisfy}(2)\right\},
$$

and $\delta_{0}$ and $c$ are given positive numbers.

We shall address another type of problems. They are quite similar to the above class. The only difference hinges on the role played by the nonlocal term $B_{0}$. We define the design problem $\left(D_{\delta}^{R}\right)$ as follows:

$$
\min _{(h, u) \in \mathcal{A}^{R}} J_{\delta}^{R},
$$

where the cost functional is

$$
J_{\delta}^{R}(h, u)=\int_{0}^{T} B(u, u) d t
$$


where $F$ is as in Section 1 and the set of admissibility is

$$
\mathcal{A}^{R}=\left\{(h, u) \in \mathcal{H}_{R}\left(\delta_{0}\right) \times Y_{0}:(h, u) \text { satisfy (2) }\right\},
$$

where

$$
\mathcal{H}_{R}\left(\delta_{0}\right)=\left\{h \in \mathcal{H}: B_{0}(h, h)<R\right\} .
$$

\subsection{2 | The classical model}

We describe the local model we are going to solve. It is indeed the corresponding regularization of (21)-(22) that will be solved by means of the minimizers of the corresponding nonlocal optimal design problem, $\left(\mathcal{D}_{\delta}\right)$ or $\left(\mathcal{D}_{\delta}^{R}\right)$.

The formulation is quite similar and the one that corresponds to the first type $\left(\mathcal{D}_{\delta}\right)$ is

$$
\left(D_{l o c}\right) \equiv \min _{(h, u) \in \mathcal{A}_{l o c}} J_{l o c}(h, u)
$$

where

$$
\begin{gathered}
J_{l o c}(h, u)=\int_{0}^{T} \int_{\Omega}|\nabla u(x, t)|^{2} d x d t+c B_{0}(h, h) \\
\mathcal{A}_{l o c}=\{(h, u) \in \mathcal{H} \times W(0, T):(h, u) \text { satisfy }(16)\} .
\end{gathered}
$$

We underline that the only difference between $\left(D_{l o c}\right)$ and $\left(D_{0}\right)$ is the perturbation implemented in cost by means of the nonlocal term $B_{0}$.

The local version of $\left(D_{\delta}^{R}\right)$ is denoted by $\left(D_{l o c}^{R}\right)$. The cost functional of $\left(D_{l o c}^{R}\right)$ is denoted by $J_{l o c}^{R}$, and it is defined as in (31) but with $c=0$. The set of admissibility of $\left(D_{l o c}^{R}\right)$ is

$$
\mathcal{A}_{\text {loc }}^{R}=\left\{(h, u) \in \mathcal{H}_{R}\left(\delta_{0}\right) \times W(0, T):(h, u) \text { satisfy }(16)\right\} .
$$

Now, the approach to $\left(D_{0}\right)$ is performed by modifying the set of admissibility. The only distinction between $\left(D_{0}\right)$ and $\left(D_{l o c}^{R}\right)$ is the nonlocal constraint $B_{0}(h, h)<R$.

\subsubsection{Concerning the bound on the controls}

It is true that the assumption made about the controls inevitably implies a certain regularity for them. If we try to solve the original problem (21)-(22), then, for a fixed small $\delta$, we should have to deal with an approximation of the solution for the nonlocal version, a sequence $\left(h_{\delta j}, u_{\delta j}\right)$. Under these circumstances, it would be interesting to analyze the evolution of the bound on the controls in terms of $j$ and $\delta$ : Compute $B_{0}\left(h_{\delta j}, h_{\delta j}\right)$ and allocate a certain positive constant $\rho$, which depends on $j$ and $\delta$, such that

$$
B_{0}\left(h_{\delta j}, h_{\delta j}\right)=\int_{\Omega_{\delta_{0}}} \int_{\Omega_{\delta_{0}}} k_{\delta_{0}}\left(\left|x^{\prime}-x\right|\right) \frac{\left|h_{\delta j}\left(x^{\prime}\right)-h_{\delta j}(x)\right|^{2}}{\left|x^{\prime}-x\right|^{2}} d x^{\prime} d x \leq \varrho(j, \delta) .
$$

The key point in order to contribute to solving the original problem (21)-(22) is the analysis of the sequence $\rho$ if we let $j \rightarrow+\infty$ with $\delta$ fixed and small. This could be a particular way to provide any sort of information concerning the behavior of the optima or about the complexity of the minimization sequences.

It is clear that $\rho$ is expected to increase if the fineness of the partition goes to zero, that is, if $j \rightarrow+\infty$. However, if we fix $\delta$ and then $j$, and for any given minimizing sequence of pairs $\left(h_{\delta j}, u_{\delta j}\right)$, we eventually compute the corresponding $\rho$, then the regularized problem (27)-(30), defined by means of $\mathcal{H}_{\rho}\left(\delta_{0}\right)$, is a finer approximation. Indeed, the solution of the latter problem $\left(h_{j}^{*}, u_{j}^{*}\right)$ is optimal because necessarily $h_{\delta j} \in \mathcal{H}_{\rho}\left(\delta_{0}\right)$, and thus, the cost achieved is below; that is,

$$
J_{\delta}\left(h_{j}^{*}, u_{j}^{*}\right) \leq J_{\delta}\left(h_{\delta j}, u_{\delta j}\right)
$$

Another ingredient we have to note, inside $B_{0}$, is the singular term $\frac{\left|h\left(x^{\prime}\right)-h(x)\right|^{2}}{\left|x^{\prime}-x\right|^{2}}$, which, somehow, can be compensated by the kernel $k_{\delta_{0}}$ if the selected $\delta_{0}$ is large enough. This factually allows us to consider wider sets of admissible controls, 
even controls with large discontinuities. Unfortunately, there is no way to pass to the limit because the assumption on the kernel made in (7).

\section{2 | G-convergence}

We consider a sequence $\left(h_{\delta}\right)_{\delta} \subset \mathcal{H}$ and $h$, its weak-* limit in $L^{\infty}(\Omega)$ as well. Let $u \in W(0, T)$ be the underlying local state of $h$, that is, the only solution of the state equation (16):

$$
\left\{\begin{array}{c}
w_{t}-\operatorname{div}(h \nabla w)=f, w \in W(0, T) \\
w(x, t)=0 \text { in } \partial \Omega \\
w(x, 0)=g(x)
\end{array}\right.
$$

We can also compute the sequence of solutions $\left(u_{\delta}\right)_{\delta}$ of the nonlocal state equation (2). Then

$$
B_{h_{\delta}}\left(u_{\delta}, w\right)=(f, w)-\left\langle\left(u_{\delta}\right)_{t}, w\right\rangle \text { for any } w \in X_{0} .
$$

If we use the notation

$$
b_{h}(u, v)=\int_{\Omega} h(x) \nabla u(x) \nabla v(x) d x
$$

then it is clear that

$$
b_{h}(u, u)=(f, u)-\left\langle u_{t}, u\right\rangle
$$

After some easy manipulations, we obtain

$$
\begin{aligned}
b_{h}(u, u) & =(f, u)-\left\langle u_{t}, u\right\rangle \\
& =\left(2 B_{h_{\delta}}\left(u_{\delta}, u\right)+2\left\langle\left(u_{\delta}\right)_{t}, u\right\rangle\right)-\left((f, u)+2\left\langle u_{t}, u\right\rangle-\left\langle u_{t}, u\right\rangle\right) \\
& =2 B_{h_{\delta}}\left(u_{\delta}, u\right)+2\left\langle\left(u_{\delta}\right)_{t}, u\right\rangle-b_{h}(u, u)-2\left\langle u_{t}, u\right\rangle \\
& =\left(2 B_{h_{\delta}}\left(u_{\delta}, u\right)-b_{h}(u, u)\right)-2\left\langle u_{t}-\left(u_{\delta}\right)_{t}, u\right\rangle .
\end{aligned}
$$

Consequently, we can write

$$
\begin{aligned}
& \lim _{\delta \rightarrow 0}\left[\left(B_{h_{\delta}}\left(u_{\delta}, u_{\delta}\right)-b_{h}(u, u)\right)-2\left\langle u_{t}-\left(u_{\delta}\right)_{t}, u\right\rangle\right] \\
= & \lim _{\delta \rightarrow 0} B_{h_{\delta}}\left(u_{\delta}-u, u_{\delta}-u\right)+\lim _{\delta \rightarrow 0}\left(b_{h}(u, u)-B_{h}(u, u)\right) \\
& +\lim _{\delta \rightarrow 0}\left(B_{h}(u, u)-B_{h_{\delta}}(u, u)\right) .
\end{aligned}
$$

In addition, we know

$$
\lim _{\delta \rightarrow 0} B_{h}(u, u)=b_{h}(u, u)
$$

and

$$
\lim _{\delta \rightarrow 0} B_{h-h_{\delta}}(u, u)=0
$$

(see Bourgain et $\mathrm{al}^{17}$ and Andrés and Muñoz ${ }^{30, \mathrm{p} 307}$ ), and therefore,

$$
\lim _{\delta \rightarrow 0}\left(B_{h_{\delta}}\left(u_{\delta}, u_{\delta}\right)+2\left\langle\left(u_{\delta}\right)_{t}, u\right\rangle\right) \geq b_{h}(u, u)+2\left\langle u_{t}, u\right\rangle
$$

We observe that $u_{\delta}$ is the solution of the problem

$$
\min _{g \in X_{0}}\left\{\frac{1}{2} B_{h_{\delta}}(g, g)-(f, g)+\left\langle\left(u_{\delta}\right)_{t}, g\right\rangle\right\}
$$

and analogously, $u$ is the solution of the problem

$$
\min _{g \in W}\left\{\frac{1}{2} b_{h}(g, g)-(f, g)+\left\langle(u)_{t}, g\right\rangle\right\} .
$$


Thus, thanks to (36), (37), and (38), we obtain the following inequality:

$$
\begin{aligned}
& \lim _{\delta \rightarrow 0} \min _{g \in X_{0}}\left\{\frac{1}{2} B_{h_{\delta}}(g, g)-(f, g)+\left\langle\left(u_{\delta}\right)_{t}, g\right\rangle\right\} \\
&=\lim _{\delta \rightarrow 0} \frac{1}{2} B_{h_{\delta}}\left(u_{\delta}, u_{\delta}\right)-\left(f, u_{\delta}\right)+\left\langle\left(u_{\delta}\right)_{t}, u_{\delta}\right\rangle \\
&=\lim _{\delta \rightarrow 0}\left(-\frac{1}{2} B_{h_{\delta}}\left(u_{\delta}, u_{\delta}\right)\right) \\
&=\lim _{\delta \rightarrow 0}\left(-\frac{1}{2} B_{h_{\delta}}\left(u_{\delta}, u_{\delta}\right)-\left\langle\left(u_{\delta}\right)_{t}, u\right\rangle+\left\langle\left(u_{\delta}\right)_{t}, u\right\rangle\right) \\
& \leq \lim _{\delta \rightarrow 0}\left[-\frac{1}{2} b_{h}(u, u)-\left\langle u_{t}, u\right\rangle+\left\langle\left(u_{\delta}\right)_{t}, u\right\rangle\right] \\
&= \lim _{\delta \rightarrow 0}\left[\frac{1}{2} b_{h}(u, u)-(f, u)+\left\langle u_{t}, u\right\rangle\right]+ \\
&+\lim _{\delta \rightarrow 0}\left\langle\left(u_{\delta}\right)_{t}-u_{t}, u\right\rangle \\
&= \min _{g \in W}\left\{\frac{1}{2} b_{h}(g, g)-(f, g)+\left\langle(u)_{t}, g\right\rangle\right\}+\lim _{\delta \rightarrow 0}\left\langle\left(u_{\delta}\right)_{t}-u_{t}, u\right\rangle .
\end{aligned}
$$

As far as the inverse inequality is concerned, it is essential to observe that the same study performed for the existence (Subsection 3.1) applies now: Since $u_{\delta}$ solves the variational formulation, then, for every $\delta$,

$$
\left\langle\left(u_{\delta}\right)_{t}, u_{\delta}\right\rangle+B_{h_{\delta}}\left(u_{\delta}, u_{\delta}\right)=\left(f, u_{\delta}\right)_{L^{2}(\Omega) \times L^{2}(\Omega)}
$$

holds. Hence, there is positive constant $C$ such that

$$
B_{h_{\delta}}\left(u_{\delta}, u_{\delta}\right) \leq C
$$

for a.e. $t$. Thus, Theorem 3 implies $u_{\delta} \rightarrow v \in H_{0}^{1}(\Omega)$ strongly in $L^{2}(\Omega)$. Under these circumstances, we have the

Proposition 2. If $h_{\delta} \rightarrow h$ a.e., then

$$
\lim _{\delta \rightarrow 0} B_{h_{\delta}}\left(u_{\delta}, u_{\delta}\right) \geq b_{h}(v, v)
$$

Proof. If we define the sequence of measures

$$
\mu_{\delta}(A)=\int_{A}\left(\int_{B(x, \delta)} k_{\delta}\left(\left|x^{\prime}-x\right|\right) \frac{\left(u_{\delta}\left(x^{\prime}\right)-u_{\delta}(x)\right)^{2}}{\left|x^{\prime}-x\right|^{2}} d x^{\prime}\right) d x
$$

for any measurable set $A \subset \Omega$, then we clearly have

$$
B_{h_{\delta}}\left(u_{\delta}, u_{\delta}\right)=\int_{\Omega} h_{\delta}(x) d \mu_{\delta}(x)
$$

Moreover,

$$
\liminf _{\delta \rightarrow 0} \mu_{\delta}(A) \geq \liminf _{\delta \rightarrow 0} \int_{A} \int_{A} k_{\delta}\left(\left|x^{\prime}-x\right|\right) \frac{\left(u_{\delta}\left(x^{\prime}\right)-u_{\delta}(x)\right)^{2}}{\left|x^{\prime}-x\right|^{2}} d x^{\prime} d x \geq \int_{A}|\nabla u(x)|^{2} d x,
$$

where the last inequality is due to a generalization of Ponce inequality (see Ponce ${ }^{19, \mathrm{p} 12}$ and Andrés and

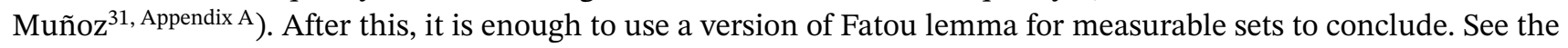
details of the proof in Andrés and Muñoz. ${ }^{31, \text { p41, Proposition } 3.2}$ 
By applying the above proposition, we get

$$
\begin{aligned}
& \lim _{\delta \rightarrow 0} \min _{g \in X_{0}}\left\{\frac{1}{2} B_{h_{\delta}}(g, g)-(f, g)+\left\langle\left(u_{\delta}\right)_{t}, g\right\rangle\right\} \\
& =\lim _{\delta \rightarrow 0} \frac{1}{2} B_{h_{\delta}}\left(u_{\delta}, u_{\delta}\right)-\lim _{\delta \rightarrow 0}\left(f, u_{\delta}\right)+\left\langle\left(u_{\delta}\right)_{t}, u_{\delta}\right\rangle \\
& \geq \frac{1}{2} b_{h}(v, v)-(f, v)+\left\langle u_{t}, v\right\rangle+\left\langle v_{t}, v\right\rangle-\left\langle u_{t}, v\right\rangle \\
& \geq \min _{g \in W}\left\{\frac{1}{2} b_{h}(g, g)-(f, g)+\left\langle u_{t}, g\right\rangle\right\}+\left\langle v_{t}-u_{t}, v\right\rangle .
\end{aligned}
$$

Then, by gathering the above estimations, we arrive at

$$
\begin{aligned}
& \min _{g \in W}\left\{\frac{1}{2} b_{h}(g, g)-(f, g)+\left\langle u_{t}, g\right\rangle\right\}+\left\langle v_{t}-u_{t}, v\right\rangle \\
\leq & \lim _{\delta \rightarrow 0} \min _{g \in X_{0}}\left\{\frac{1}{2} B_{h_{\delta}}(g, g)-(f, g)+\left\langle\left(u_{\delta}\right)_{t}, g\right\rangle\right\} \\
\leq & \min _{g \in W}\left\{\frac{1}{2} b_{h}(g, g)-(f, g)+\left\langle(u)_{t}, g\right\rangle\right\}+\lim _{\delta \rightarrow 0}\left\langle\left(u_{\delta}\right)_{t}-u_{t}, u\right\rangle \\
= & \min _{g \in W}\left\{\frac{1}{2} b_{h}(g, g)-(f, g)+\left\langle(u)_{t}, g\right\rangle\right\}+\left\langle v_{t}-u_{t}, u\right\rangle .
\end{aligned}
$$

Then

and so, for any $s \in(0, T)$,

$$
\left\langle v_{t}-u_{t}, v-u\right\rangle \leq 0,
$$

Thus,

$$
\int_{0}^{s} \frac{1}{2} \frac{d}{d t}\left(\|v(t)-u(t)\|_{L^{2}}^{2}\right) d t \leq 0
$$

which amounts to say $u=v$ a.e. Moreover, from (39), we get

$$
\|v(s)-u(s)\|_{L^{2}}^{2} \leq 0,
$$

$$
\lim _{\delta \rightarrow 0} B_{h_{\delta}}\left(u_{\delta}, u_{\delta}\right)=b_{h}(u, u)
$$

By taking into account that

$$
\begin{aligned}
\lim _{\delta \rightarrow 0} B_{h_{\delta}}\left(u_{\delta}, u\right) & =\lim _{\delta \rightarrow 0}\left((f, u)-\left\langle\left(u_{\delta}\right)_{t}, u\right\rangle\right) \\
& =(f, u)-\left\langle u_{t}, u\right\rangle \\
& =b_{h}(u, u),
\end{aligned}
$$

it is strightforward to check the following strong convergence in $X_{0}$ :

$$
\lim _{\delta \rightarrow 0} B_{h_{\delta}}\left(u_{\delta}-u, u_{\delta}-u\right)=0
$$

The above limit and the dominated convergence theorem provide strong convergence of $\left(u_{\delta}\right)_{\delta}$ (at least for a subsequence) to $u$ in $Y_{0}$, in the sense that

We have proved the following result:

$$
\lim _{\delta \rightarrow 0}\left\|u_{\delta}-u\right\|_{Y_{0}}=0
$$

Theorem 5. If $\left(h_{\delta}\right)_{\delta} \subset \mathcal{H}$ and $h_{\delta} \rightarrow$ ha.e. $x \in \Omega$, then

$$
\begin{aligned}
& \lim _{\delta \rightarrow 0} \min _{g \in X_{0}}\left\{\frac{1}{2} B_{h_{\delta}}(g, g)-(f, g)+\left\langle\left(u_{\delta}\right)_{t}, g\right\rangle\right\} \\
& =\min _{g \in W}\left\{\frac{1}{2} b_{h}(g, g)-(f, g)+\left\langle(u)_{t}, g\right\rangle\right\}
\end{aligned}
$$

and $u_{\delta} \rightarrow u$ strongly both in $L^{2}$ and also in $Y_{0}$ in the sense described above. 


\subsection{The local optimal design problem as the limit when $\delta \rightarrow 0$}

We analyze the limit of the solutions of the problem $\left(\mathcal{D}_{\delta}\right)$. Let $\left(h_{\delta}, u_{\delta}\right)$ be the sequence of solutions of $\left(\mathcal{D}_{\delta}\right)$. We know $h_{\delta} \rightarrow h$ weak-* in $L^{\infty}$.

We notice

$$
J_{\delta}\left(h_{\delta}, u_{\delta}\right) \leq J_{\delta}\left(h^{*}, u_{\delta}^{*}\right)
$$

for any regular admissible $h^{*} \in \mathcal{H}$ whose associated state is $u_{\delta}^{*}$. Then $u_{\delta}^{*} \rightarrow u^{*} \in H^{1}(\Omega)$ so that

$$
\lim _{\delta \rightarrow 0} B_{h^{*}}\left(u_{\delta}^{*}, u_{\delta}^{*}\right)=b_{h^{*}}\left(u^{*}, u^{*}\right)
$$

this fact implies $B\left(u_{\delta}^{*}, u_{\delta}^{*}\right)$ is uniformly bounded. By noting that

$$
J_{\delta}\left(h^{*}, u_{\delta}^{*}\right)=\int_{0}^{T} B\left(u_{\delta}^{*}, u_{\delta}^{*}\right) d t+c B_{0}\left(h^{*}, h^{*}\right)
$$

and taking into account that $B_{0}\left(h^{*}, h^{*}\right)$ is finite, we deduce $J_{\delta}\left(h_{\delta}, u_{\delta}\right)$ is uniformly bounded. This fact, in particular, ensures $B_{0}\left(h_{\delta}, h_{\delta}\right)$ is uniformly bounded too. That means the sequence $\left(h_{\delta}\right)_{\delta}$ is included in the set $\mathcal{H}_{R}\left(\delta_{0}\right)$ for some $R>0$. Since $\mathcal{H}_{R}\left(\delta_{0}\right)$ is compactly imbedded in $L^{2}$ (see Theorem 2 or see the details in Andrés and Muñoz ${ }^{31}$, p51), then there exists a subsequence of $\left(h_{\delta}\right)_{\delta}$, which will be also denoted by $\left(h_{\delta}\right)_{\delta}$, that converges to the function $h$ strongly in $L^{2}(\Omega)$.

Concerning the states the analysis of the previous sections ensures the sequence $\left(u_{\delta}\right)_{\delta}$ (or a subsequence of it) converges strongly in $L^{2}$ to a function $u \in H_{0}^{1}(\Omega)$.

Consequently, Theorem 5 states the pair $(h, u) \in \mathcal{A}_{\text {loc }}$.

Next, we analyze the optimality of $(h, u)$. In order to do that we consider $\left(\tilde{u}_{\delta}\right)_{\delta}$ the sequence of solutions for the nonlocal state Equation 16 when we take $h$ as design. Then $\left(h, \tilde{u}_{\delta}\right) \in \mathcal{A}$. By using Andrés and Muñoz, ${ }^{42, \text { Th. }}{ }^{1.4}$ we can assert that

$$
\lim _{\delta \rightarrow 0} B_{h}\left(\tilde{u}_{\delta}-u, \tilde{u}_{\delta}-u\right)=0
$$

Moreover,

$$
\lim _{\delta \rightarrow 0} B_{h}\left(\tilde{u}_{\delta}, \tilde{u}_{\delta}\right)=\lim _{\delta \rightarrow 0} B_{h}(u, u)=\int_{\Omega}|\nabla u(x, t)|^{2} d x .
$$

By using this identity and the dominated convergence theorem, we obtain

$$
\begin{aligned}
\lim _{\delta \rightarrow 0} J_{\delta}\left(h_{\delta}, u_{\delta}\right) & \leq \lim _{\delta \rightarrow 0} J_{\delta}\left(h, \tilde{u}_{\delta}\right) \\
& =\lim _{\delta \rightarrow 0} \int_{0}^{T} B\left(\tilde{u}_{\delta}, \tilde{u}_{\delta}\right) d t+c B_{0}(h, h) \\
& =\int_{0}^{T} \int_{\Omega}|\nabla u(x, t)|^{2} d x d t+c B_{0}(h, h) \\
& =J_{l o c}(h, u) .
\end{aligned}
$$

For the reverse estimation, we use Ponce inequality (see Ponce ${ }^{19}$ and Andrés and Muñoz ${ }^{31, \text { Appendix A, p48 }}$ ); that is, for any $t \in(0, T)$,

$$
\lim _{\delta \rightarrow 0} B\left(u_{\delta}, u_{\delta}\right) \geq \int_{\Omega}|\nabla u(x, t)|^{2} d x .
$$

By means of the above inequality and by Fatou Lemma to arrive at

$$
\begin{aligned}
\lim _{\delta \rightarrow 0} J_{\delta}\left(h_{\delta}, u_{\delta}\right) & =\lim _{\delta \rightarrow 0} \int_{0}^{T} B\left(u_{\delta}, u_{\delta}\right) d t+c \lim _{\delta \rightarrow 0} B_{0}\left(h_{\delta}, h_{\delta}\right) \\
& \geq \int_{0}^{T} \int_{\Omega}|\nabla u(x, t)|^{2} d x d t+c \lim _{\delta \rightarrow 0} B_{0}(h, h) \\
& =J_{l o c}(h, u) .
\end{aligned}
$$


Thus, we have proved

$$
\lim _{\delta \rightarrow 0} J_{\delta}\left(h_{\delta}, u_{\delta}\right)=J_{l o c}(h, u) .
$$

Also, it is automatic to check that if $\left(h^{*}, u^{*}\right) \in \mathcal{A}_{l o c}$ and $u_{\delta}^{*}$ is the underlying state of $h^{*}$ then, as above, $u_{\delta}^{*} \rightarrow u^{*}$ in $L^{2}$ and

$$
\begin{aligned}
\lim _{\delta \rightarrow 0} J_{\delta}\left(h_{\delta}, u_{\delta}\right) & \leq \lim _{\delta \rightarrow 0} J_{\delta}\left(h^{*}, u_{\delta}^{*}\right) \\
& =J_{l o c}\left(h^{*}, u^{*}\right) .
\end{aligned}
$$

Therefore,

$$
J_{l o c}(h, u) \leq J_{l o c}\left(h^{*}, u^{*}\right)
$$

for any $\left(h^{*}, u^{*}\right) \in \mathcal{A}_{l o c}$, namely, $(h, u)$ is optimal for the problem $\left(D_{l o c}\right)$. This completes the proof of the

Theorem 6. Let $\left(h_{\delta}\right)_{\delta}$ be a sequence of optimal designs for the problems $\left(\mathcal{D}_{\delta}\right)$ and let $\left(u_{\delta}\right)_{\delta}$ be their underlying states.

Then there exists a pair $(h, u) \in \mathcal{A}_{\text {loc }}$ and a subsequence of $\delta^{\prime}$ s such that

1.

$$
\begin{aligned}
& h_{\delta} \rightarrow \text { h a.e. in } \Omega, \\
& u_{\delta} \rightarrow u \text { strongly in } L^{2}(\Omega) .
\end{aligned}
$$

2.

$$
\lim _{\delta \rightarrow 0} J_{\delta}\left(h_{\delta}, u_{\delta}\right)=J_{l o c}(h, u)
$$

3. The pair $(h, u)$ is a solution of the local design problem ( $\left.D_{\text {loc }}\right)$ defined in (31)-(33).

The same approach serves to approximate several types of problems of optimization. For instance, Theorem 6 applies to problems like

or

$$
J_{l o c}(h, u)=\alpha \int_{0}^{T}\left(B(u, u)+\|u\|_{L^{2}(\Omega)}^{2}\right) d t+\beta B_{0}(h, h)
$$

$$
J_{l o c}(h, u)=\alpha \int_{0}^{T}(B(u, u)+g u) d t+\beta B_{0}(h, h)
$$

with $(h, u) \in \mathcal{A}_{l o c}$. Here, $\alpha$ and $\beta$ are given positive constants and $g$ is any function from $L^{2}\left((0, T) ; L^{2}(\Omega)\right)$.

We can generalize Theorem 6 to problems under the format

$$
J_{l o c}(h, u)=\alpha \int_{0}^{T} B(u, u) d t+\int_{0}^{T} \int_{\Omega} G(x, u) d x d t+\beta B_{0}(h, h)
$$

where $G: \Omega \times \mathbb{R} \rightarrow \mathbb{R}$ is any measurable function such that $G(x, \cdot)$ is uniformly Lipschitz continuous.

Concerning the type of problems $\left(D_{l o c}^{R}\right)$, the outcome we obtain is identical to the above case. We have the following:

Theorem 7. If $\left(h_{\delta}, u_{\delta}\right)$ is a sequence of solutions for the problem $\left(D_{\delta}^{R}\right)$, then we can find $(h, u) \in \mathcal{A}_{\text {loc }}^{R}$ and a subsequence of $\delta$ 's such that

1. $h_{\delta} \rightarrow$ h a.e. in $\Omega, u_{\delta} \rightarrow u$ strongly in $L^{2}(\Omega)$

2.

$$
\lim _{\delta \rightarrow 0} J_{\delta}^{R}\left(h_{\delta}, u_{\delta}\right)=J_{l o c}^{R}(h, u) .
$$

3. $(h, u)$ is a minimizer of the problem $\left(D_{l o c}^{R}\right)$.

Concerning the generalization of this theorem, the same comments of the problem $\left(D_{l o c}\right)$ applies in the present setting.

\subsection{Conclusions}

At first sight, the above discussion gives an explicit answer about how to solve or approximate some classical optimal design problems. Despite these results concern a matter that, by itself, is of great importance, our main goal is to give some information about the minimizers or minimizing sequences of the problem $\left(D_{0}\right)$. This goal has only been partially achieved. Two alternatives have been explored: 
1. One approach in dealing with $\left(D_{0}\right)$ relies on a penalization of the cost functional. This has been done in $\left(\mathcal{D}_{\delta}\right)$. In the limit, $\left(\mathcal{D}_{\delta}\right)$ has given rise to $\left(D_{l o c}\right)$, which is, in a certain sense, a regularization of the original problem $\left(D_{0}\right)$. Consequently, it seems appropriate to simulate the solutions of $\left(\mathcal{D}_{\delta}\right)$ when the coefficient of the perturbation $c$ tends to 0 . This a method that could allow us to detect meaningful information about the minimization problem $\left(D_{0}\right)$

2. The second way to tackle $\left(D_{0}\right)$ is by means a modification of its set of admissibility. This is achieved by formulating $\left(D_{\delta}^{R}\right)$ and analyzing the limit of its solution. The resultant problem, $\left(D_{l o c}^{R}\right)$, is somehow a regularization of $\left(D_{0}\right)$. Thus, the analysis of the solutions of $\left(D_{\delta}^{R}\right)$ when $R \rightarrow+\infty$ could provide interesting information about the optimization of $\left(D_{0}\right)$.

In either case, the numerical simulations performed under these type of hypotheses on the control and the comparison to existing computations from the bibliography could help in going a step further about the idea of regularization we proposed at the beginning of the paper.

\section{ACKNOWLEDGEMENTS}

This paper was supported by Project MTM2017-87912-P, Ministerio de Economía, Industria y Competitividad (Spain). The authors are grateful to an unknown referee for interesting suggestions to improve the readability of the paper. There are no conflicts of interest to this work

\section{ORCID}

Julio Muñoz (DD https://orcid.org/0000-0002-9964-2358

\section{REFERENCES}

1. Bucur C, Valdinoci E. Nonlocal Diffusion and Applications, Lecture Notes of the Unione Matematica Italiana, vol. 20. Bologna: Springer. Unione Matematica Italiana; 2016.

2. Vázquez JL. The Mathematical theories of diffusion: nonlinear and fractional diffusion. In: Bonforte M, Grillo G, eds. Nonlocal and Nonlinear Diffusions and Interactions: New Methods and Directions, Lecture Notes in Mathematics, vol. 2186. Cham: Springer; 2017:205-278.

3. Levendorski SZ. Pricing of the American put under Levy processes. Int J Theor Appl Finance. 2014;7:303-335.

4. Bakunin O. Turbulence and Diffusion: Scaling Versus Equations. Berlin, Heidelberg: Springer Verlag; 2008.

5. Constantin P. Euler equations, Navier-Stokes equations and turbulence. Mathematical foundation of turbulent viscous flows, Lecture Notes in Math. Berlin: Springer; 2006:1-43.

6. Alali B, Lipton R. Multiscale dynamics of heterogeneous media in the peridynamic formulation. J Elasticity. 2012;106:71-103.

7. Silling SA. Reformulation of elasticity theory for discontinuities and long-range forces. J Mech Phys Solids. 2000;48:175-209.

8. Silling SA, Epton M, Weckner O, Xu J, Askari E. Peridiynamics states and constitutive modelling. J Elasticity. 2007;88:151-184.

9. Hutson V, Martínez S, Michaidow K, Vickers GT. The evolution of dispersal. J Math Biol. 2003;47(6):483-517.

10. Humphries NE, Queiroz N, Dyer JR, et al. Environmental context explains Lévy and Brownian movement patterns of marine predators. Nature. 2010;465:1066-1069.

11. Gilboa G, Osher S. Nonlocal operators with applications to image processing. Multiscale Model Simul. 2008;7:1005-1028.

12. Lou Y, Zhang X, Osher S, Bertozzi A. Image recovery via nonlocal operators. J Sci Comput. 2010;42:185-197.

13. Bazant ZP, Jirasek M. Nonlocal integral formulation of plasticity and damage: survey of progress. J Engrg Mech. 2002;128:1119-1149.

14. Brandon D, Rogers RC. Nonlocal regularization of L. C. Young's tacking problem. Appl Math Optim. 1992;25(3):287-301. https://doi.org/ 10.1007/BF01182325

15. Rogers RC. A nonlocal model for the exchange energy in ferromagnetic materials. J Integral Equations Appl. 1991;3:85-127.

16. Andreu-Vaillo F, Mazón JM, Rossi JD, Toledo-Melero JJ. Nonlocal Diffusion problems, Mathematical Surveys and Monographs, vol. 165. Providence, R.I.: American mathematical society; 2010.

17. Bourgain J, Brezis H, Mironescu P. Another look at Sobolev spaces. In: Menaldi JL et al., eds. Optimal Control and Partial Differential Equations. Amsterdam: IOS Press; 2001:439-455. (A volume in honour of A. Benssoussan's 60th birthday).

18. Brezis H. How to recognize constant functions. Connections with Sobolev spaces. Uspekhi Mat Nauk. 2002;57(4(346)):59-74.

19. Ponce AC. An estimate in the spirit of Poincaré's inequality. J Eur Math Soc (JEMS). 2004;6:1-15.

20. Ponce AC. A new approach to Sobolev spaces and connections to $\Gamma$-convergence. Calc Var. 2004;19:229-255.

21. Caffarelli LA. Non-local diffusions, drifts and games. Nonlinear Partial Differential Equations, Abel Symp., vol. 7. Heidelberg: Springer; 2012:37-52.

22. Vázquez JL. Asymptotic behaviour methods for the heat equation. Convergence to the Gaussian. arXiv:1706.10034 [math.AP]; 2018.

23. D'Elia M, Perego M, Bochev P, Littlewood D. A coupling strategy for nonlocal and local diffusion models with mixed volume constraints and boundary conditions. Computers \& Mathematics with Applications. 2016;71(11):2218-2230. 
24. D'Elia M, Gunzburger M. The fractional Laplacian operator on bounded domains as a special case of the nonlocal diffusion operator. Comp Math Appl. 2013;66:1245-1260.

25. Guan Q, Gunzburger M. Stability and convergence of time-stepping methods for a nonlocal model for diffusion. Disc Cont Dynam Sys-B. 2015;20(5):1315-1335.

26. Guan Q, Gunzburger M. Stability and accuracy of time-stepping schemes and dispersion relations for a nonlocal wave equation. Numer Methods Partial Differential Eq. 2015;31:500-516.

27. Puhst DD. On the evolutionary fractional p-Laplacian. Appl Math Res Express. 2015;2:253-273.

28. Seleson P, Gunzburger M, Parks M. Interface problems in nonlocal diffusion and sharp transitions between local and nonlocal domains. Comput Meth Appl Mech Enrgr. 2013;266:185-204.

29. Seleson P, Parks M, Gunzburger M. Peridynamic solid mechanics and the embedded-atom model. Comm Comput Phys. 2014;15:179-205.

30. Andrés F, Muñoz J. Nonlocal optimal design: a new perspective about the approximation ofsolutions in optimal design. J Math Anal Appl. 2015;429:288-310.

31. Andrés F, Muñoz J. On the convergence of a class of nonlocal elliptic equations and related optimal design problems. J Optim Theory Appl. 2016;172:33-55. https://doi.org/10.1007/s10957-016-1021-z

32. Bonder JF, Spedaletti JF. Some nonlocal optimal design problems. J Math Anal Appl. 2018;459(2):906-931.

33. D'Elia M, Gunzburger M. Optimal distributed control of nonlocal steady diffusion problems. SIAM J Control Optim. 2014;52(1):243-273.

34. D'Elia M, Gunzburger M. Identification of the diffusion parameter in nonlocal steady diffusion problems. Appl Math Optim. 2016;73(2):227-249.

35. Zhou K, Du Q. Mathematical and numerical analysis of linear peridynamic models with nonlocal boundary conditions. SIAM J Numer Anal. 2010;48(5):1759-1780.

36. Zhou K, Du Q. Mathematical analysis for the peridynamic nonlocal continuum theory. ESAIM: Math Mod Num Anal. 2011;45:217-234.

37. Bonder JF, Ritorto A, Martín A. H-convergence result for nonlocal elliptic-type problems via Tartar's method. SIAM J Math Anal. 2017;49(4):2387-2408.

38. Andrés F. Aproximación Y Optimización De Problemas No Locales. Memoria Tesis Doctoral: Escuela de Ingenieros de Toledo Departamento de Matemáticas de la Universidad de Castilla-La Mancha; 2016.

39. Hunter JK. Notes on partial differential equations. Manuscript. https://www.math.ucdavis.edu/ hunter/pdes/pde_notes.pdf

40. Tröltzsch F. Optimal Control of Partial Differential Equations, Theory, Methods and Applications. Providence, R.I: American Math. Soc.; 2010.

41. Wloka J. Partial Differential Equations. Cambridge: Cambridge University Press; 1987.

42. Andrés F, Muñoz J. A type of nonlocal elliptic problem: existence and approximation through a Galerkin-Fourier method. SIAM J Math Anal. 2015;47(1):498-525.

43. Andrés F, Muñoz J. The Galerkin-Fourier method for the study of nonlocal parabolic equations. Z. Angew. Math. Phys. 2019;70:92. https:// doi.org/10.1007/s00033-019-1136-9

44. Servadei R, Valdinoci E. Variational methods for non-local operators of elliptic type. Discrete Contin Dyn Syst. 2013;33:2105-2137.

45. Du Q, Gunzburger M, Lehoucq R, Zhou K. Analysis and approximation of nonlocal diffusion problems with volume constraints. SIAM Rev. 2012;54(4):667-696.

46. Mengesha T, Du Q. On the variational limit of a class of nonlocal functionla related to peridynamics. Nonlinearity. 2105;28(11):3999-4036.

47. Lions JL. Contrôle Optimal de Systèmes Gouvernes par Des Équations Aux Dérivées partielles. Paris: Dunod-Guthier-Villars; 1968.

48. Evans LC. Partial Differential Equations. Providence: American Math. Soc.; 1999.

49. Lions JL, Magenes E. Problèmes Aux Limites Non Homogénes et Applications. Paris: 1, 2, 3, Dunod; 1968.

How to cite this article: Andrés F, Muñoz J, Rosado J. Existence and approximation of nonlocal optimal design problems driven by parabolic equations. Math Meth Appl Sci. 2019;1-18. https://doi.org/10.1002/mma.5701

\section{APPENDIX A}

Theorem 8 (see previous works $\left.{ }^{39-41,49}\right)$. Let $V \subset H \subset V^{\prime}$ be a Gelfand triplet. If $u \in L^{2}(0, T, V)$ and $u_{t} \in L^{2}\left(0, T, V^{\prime}\right)$ then $C([0, T] ; H)$. Moreover,

1. For any $v \in V$, the real-valued function $t \rightarrow(u, v)_{H}$ is weakly differentiable in $(0, T)$ and

$$
\frac{d}{d t}(u, v)_{H}=\left\langle u_{t}, v\right\rangle
$$


2. The real-valued function $t \rightarrow\|u(t)\|_{L^{2}\left(\Omega_{\delta}\right)}^{2}$ is weakly differentiable in $(0, T)$ and

$$
\frac{1}{2} \frac{d}{d t}\left(\|u(t)\|_{H}^{2}\right)=\left\langle u_{t}(t), u(t)\right\rangle .
$$

3. There is a constant $C=C(T)$ such that

$$
\|u(t)\|_{L^{\infty}(0, T ; H)} \leq C\left(\|u(t)\|_{L^{2}(0, T ; V)}+\left\|u_{t}\right\|_{L^{2}\left(0, T ; V^{\prime}\right)}\right) .
$$

